\title{
Correction: A bibliometric analysis of the interdisciplinary field of cultural evolution
}

\author{
Mason Youngblood (10 ${ }^{1,2} \&$ David Lahti ${ }^{1,2}$
}

Correction to: Palgrave Communications https://doi.org/10.1057/s41599-018-0175-8, Published online 2 Oct 2018

In the original version of the manuscript the legend of Fig. 2 was ambiguous as to how individuals were excluded from the network to maximize legibility, potentially leading to the perception that it may have been a subjective decision on the authors' part. As such, a higher resolution image has been included in Fig. 2, and additional detail in the legend. These changes do not reflect a change in the study's underlying data or results.

The image below is now displayed in Fig. 2 .

The following change is made the legend of Fig. 2:

Original:

The largest connected co-authorship network in the dataset, analyzed using VOS clustering $(n=629)$. Red corresponds to group 1 ("biological anthropology and archeology"; $n 1=183$ ), green corresponds to group 2 ("mathematical modeling and dual-inheritance theory"; $n 2=146$ ), blue corresponds to group 3 ("cognitive linguistics and experimental cultural evolution"; $n 3=134$ ), yellow corresponds to group 4 ("cross-cultural and phylogenetic studies"; $n 4=75$ ), purple corresponds to group 5 ("computational biology and cultural niche construction"; $n 5=56$ ), cyan corresponds to group 6 ("evolutionary psychology"; $n 6=20$ ), and orange corresponds to group 7 ("behavioral ecology and birdsong"; $n 7=15$ ). Name size indicates total link strength, and the authors are subsequently filtered by VOSviewer to maximize legibility.

Revised:

The largest connected co-authorship network in the dataset, analyzed using VOS clustering $(n=629)$. Red corresponds to group 1 ("biological anthropology and archeology"; $n 1=183$ ), green corresponds to group 2 ("mathematical modeling and dual-inheritance theory"; $n 2=146$ ), blue corresponds to group 3 ("cognitive linguistics and experimental cultural evolution"; $n 3=134$ ), yellow corresponds to group 4 ("cross-cultural and phylogenetic studies"; $n 4=75$ ), purple corresponds to group 5 ("computational biology and cultural niche construction"; $n 5=56$ ), cyan corresponds to group 6 ("evolutionary psychology"; $n 6=20$ ), and orange corresponds to group 7 ("behavioral ecology and birdsong"; $n 7=15$ ). Name size indicates total link strength. Many authors were arbitrarily excluded from the figure by the visualization algorithm in VOSviewer to maximize legibility. A complete, interactive version of the network can be found in the Dataverse repository entry: https://doi.org/10.7910/DVN/LBIDEL

In addition, the original version of this paper omitted an acknowledgement of the research funder. The following acknowledgement is now included in the paper:

Funding was provided by the John Templeton Foundation Grant ID 60459.

\footnotetext{
${ }^{1}$ Department of Psychology, The Graduate Center, City University of New York, New York, NY, USA. ${ }^{2}$ Department of Biology, Queens College, City University of New York, Flushing, NY, USA. Correspondence and requests for materials should be addressed to

M.Y. (email: myoungblood@gradcenter.cuny.edu)
} 
The article has been corrected online.

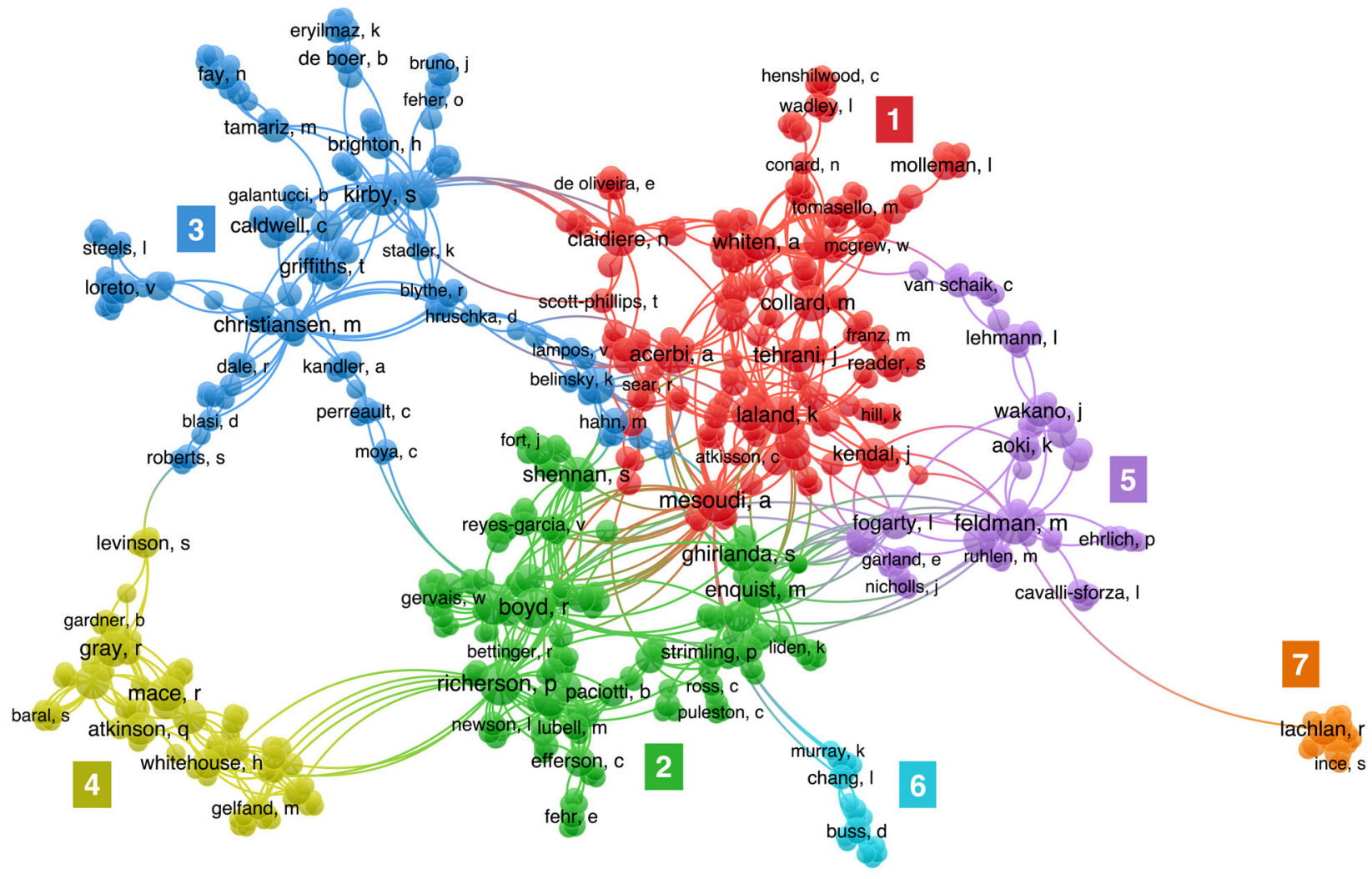

Published online: 30 October 2018

(c) Open Access This article is licensed under a Creative Commons Attribution 4.0 International License, which permits use, sharing, adaptation, distribution and reproduction in any medium or format, as long as you give appropriate credit to the original author(s) and the source, provide a link to the Creative Commons license, and indicate if changes were made. The images or other third party material in this article are included in the article's Creative Commons license, unless indicated otherwise in a credit

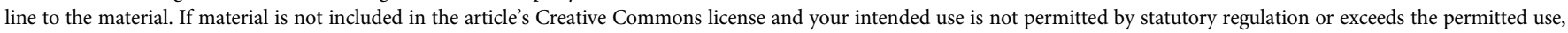
you will need to obtain permission directly from the copyright holder. To view a copy of this license, visit http://creativecommons.org/licenses/by/4.0/.

(C) The Author(s) 2018 